\title{
PROJECTIVE INTEGRAL INVARIANTS ATTACHED TO THE TRAJECTORIES OF DIFFERENTIAL SYSTEMS*
}

BY A. D. MICHAL

1. Introduction. In his book on integral invariants, E. Car$\tan \dagger$ showed how to every integral invariant in the sense of Poincaré there corresponds a more general integral invariant (called complete by Cartan) in an associated manifold of one more dimension. It turns out, as Goursat $\ddagger$ has more recently shown, that a complete integral invariant is a Poincaré integral invariant attached to the trajectories of an associated differential system.

Cartan, however, did not give necessary and sufficient conditions; neither did he go into the question of the existence of other integral invariants in the same associated manifold. The present paper is concerned with such problems. As in my previous contributions $\S$ to the subject of integral invariants, the tensor methods and the language of group theory will be employed throughout the paper.

2. Projective Transformations of Coordinates and Projective Tensors. In this paragraph and throughout our whole paper we shall understand that a Greek index can take on any of the values $0,1,2, \cdots, n$, while a Latin index can take on any of the values 1 , $2, \cdots, n$. The repetition of an index in a term will be used to denote summation with respect to that index over all its admissible values.

Let $\xi^{i}\left(x^{1}, \cdots, x^{n}\right)$ be a contravariant vector in an $n$-dimensional manifold $\left(x^{1}, x^{2}, \cdots, x^{n}\right)$. Consider an associated $(n+1)$-dimensional manifold with points having coordinates $\left(x^{0}, x^{1}, \cdots, x^{n}\right)$ and subject to the analytic transformations

$$
\text { (s): } \begin{aligned}
& \bar{x}^{i}=f^{i}\left(x^{1}, x^{2}, \cdots, x^{n}\right), \\
& \bar{x}^{0}=x^{0}+f^{0}\left(x^{1}, x^{2}, \cdots, x^{n}\right),
\end{aligned}\left|\frac{\partial f^{i}}{\partial x^{j}}\right| \neq 0,
$$

\footnotetext{
* Presented to the Society, November 29, 1930.

$\dagger$ E. Cartan, Leçons sur les Invariants Intégraux, 1922.

¥ E. Goursat, Comptes Rendus, vol. 174 (1922), pp. 1089-1091.

$\S$ A. D. Michal, Transactions of this Society, vol. 29 (1927), pp. 612-646.
} 
where $f^{0}\left(x^{1}, \cdots, x^{n}\right)$ satisfies the differential equation

$$
\xi^{i} \frac{\partial f}{\partial x^{i}}=0
$$

Theorem 1. The differential system

$$
\frac{d x^{\alpha}}{d \tau}=\xi^{\alpha}\left(x^{1}, \cdots, x^{n}\right), \quad\left(\xi^{0} \equiv 1\right),
$$

remains invariant in form under the coordinate transformations (5) in $x^{0}, x^{1}, \cdots, x^{n}$.

In fact the functions $\bar{\xi}^{\alpha}$ in the transformed equations

are given by

$$
\frac{d \bar{x}^{\alpha}}{d \tau}=\bar{\xi}^{\alpha}
$$

But

$$
\left\{\begin{array}{l}
\bar{\xi}^{i}=\xi^{j} \frac{\partial \bar{x}^{i}}{\partial x^{j}}+\frac{\partial \bar{x}^{i}}{\partial x^{0}} \\
\bar{\xi}^{0}=\xi^{j} \frac{\partial \bar{x}^{0}}{\partial x^{j}}+\xi^{0} \frac{\partial \bar{x}^{0}}{\partial x^{0}}
\end{array}\right.
$$

$$
\frac{\partial \bar{x}^{i}}{\partial x^{0}}=0, \quad \frac{\partial \bar{x}^{0}}{\partial x^{0}}=1 \text { and } \xi^{j} \frac{\partial \bar{x}^{0}}{\partial x^{j}}=0 .
$$

The truth of the theorem is now immediate.

One can also verify that the transformations \& are the most general coordinate transformations in $x^{0}, x^{1}, \cdots, x^{n}$ that leave the differential equations (3) invariant in form.

We shall use the term projective tensor* to denote a tensor whose components depend on $x^{1}, x^{2}, \cdots, x^{n}$ but not on $x^{0}$ and which is subject to the coordinate transformations (\$). It is obvious from the definition that the transformed components of a projective tensor depend only on $\bar{x}^{1}, \bar{x}^{2}, \cdots, \bar{x}^{n}$ and not on $\bar{x}^{0}$. As examples we may take the projective contravariant and covariant vectors

* Compare with T. Y. Thomas' use of the same terminology in another connection. See his paper in Mathematische Zeitschrift, vol. 25 (1926), pp. 723733. 


$$
\begin{aligned}
& \bar{A}^{\alpha}\left(\bar{x}^{1}, \cdots, \bar{x}^{n}\right)=A^{\sigma}\left(x^{1}, \cdots, x^{n}\right) \frac{\partial \bar{x}^{\alpha}}{\partial x^{\sigma}} \rightarrow\left\{\begin{array}{l}
\bar{A}^{i}=A^{j} \frac{\partial \bar{x}^{i}}{\partial x^{j}}, \\
\bar{A}^{0}=A^{j} \frac{\partial \bar{x}^{0}}{\partial x^{j}}+A^{0} ;
\end{array}\right. \\
& \bar{A}_{\alpha}\left(\bar{x}^{1}, \cdots, \bar{x}^{n}\right)=A_{\sigma}\left(x^{1}, \cdots, x^{n}\right) \frac{\partial x^{\sigma}}{\partial \bar{x}^{\alpha}} \rightarrow\left\{\begin{array}{l}
\bar{A}_{i}=A_{j} \frac{\partial x^{j}}{\partial \bar{x}^{i}}+A_{0} \frac{\partial x^{0}}{\partial \bar{x}^{i}} \\
\bar{A}_{0}=A_{0} .
\end{array}\right.
\end{aligned}
$$

3. Some Preliminary Theorems on Integral Invariants. I shall summarize in this paragraph a few theorems that will be found useful in the succeeding paragraphs. These theorems are special cases of some more general theorems proved by me in another paper.*

THEOREM 2. A necessary and sufficient condition that

$$
A_{i} \delta x^{i}
$$

be an integral invariant of the one-parameter group

$$
\frac{d x^{i}}{d \tau}=\xi^{2}\left(x^{1}, \cdots, x^{n}\right)
$$

is that the functions $A_{i}\left(x^{1}, \cdots, x^{n}\right)$ satisfy the system of tensor differential equations

$$
\left(\frac{\partial A_{i}}{\partial x^{j}}-\frac{\partial A_{j}}{\partial x^{i}}\right) \xi^{j}+\frac{\partial\left(A_{j} \xi^{j}\right)}{\partial x^{i}}=0 .
$$

The corresponding tensor differential equations for the invariance of the alternating form

$$
A_{i j}\left(x^{1}, \cdots, x^{n}\right) \delta_{1} x^{i} \delta_{2} x^{i} \quad\left(A_{i j}=-A_{j i}\right)
$$

are given by

(9) $\left(\frac{\partial A_{i j}}{\partial x^{k}}-\frac{\partial A_{k j}}{\partial x^{i}}-\frac{\partial A_{i k}}{\partial x^{j}}\right) \xi^{k}+\frac{\partial\left(A_{i l} \xi^{l}\right)}{\partial x^{j}}-\frac{\partial\left(\dot{A}_{j l} \xi^{l}\right)}{\partial x^{i}}=0$.

THEOREM 3. A necessary and sufficient condition that (5) be an integral invariant that is attached to the path curves of the group (6) is that the functions $A_{i}$ satisfy the tensor equations

* A. D. Michal, loc. cit.

$\dagger$ E. Goursat, Leçons sur le Probleme de Pfaff, 1922, p. 236. 


$$
\left(\frac{\partial A_{i}}{\partial x^{j}}-\frac{\partial A_{j}}{\partial x^{i}}\right) \xi^{j}=0, A_{i} \xi^{i}=0
$$

The corresponding tensor equations for the form (8) are

$$
\left(\frac{\partial A_{i j}}{\partial x^{k}}-\frac{\partial A_{k j}}{\partial x^{i}}-\frac{\partial A_{i k}}{\partial x^{j}}\right) \xi^{k}=0, A_{i j} \xi^{j}=0 .
$$

4. Linear Integral Invariants. One can easily show that a necessary and sufficient condition that a differential form

$$
A_{\alpha}\left(x^{1}, \cdots, x^{n}\right) \delta x^{\alpha}
$$

be an absolute form under the coordinate transformations $B$ is that $A_{0}, A_{1}, \cdots, A_{n}$ be the components of a projective covariant vector. This is the reason why we shall call the differential form (12) a projective differential form. Similar remarks hold good for the projective differential form

$$
\begin{aligned}
& A_{\alpha \beta} \delta_{1} x^{\alpha} \delta_{2} x^{\beta} \quad\left(A_{\alpha \beta}=-A_{\beta \alpha}\right),
\end{aligned}
$$

Theorem 4. A necessary and sufficient condition that the projective differential form

$$
A_{\alpha}\left(x^{1}, \cdots, x^{n}\right) \delta x^{\alpha}
$$

be an invariant of the group

$$
\frac{d x^{\alpha}}{d \tau}=\xi^{\alpha}\left(x^{1}, \cdots, x^{n}\right) \quad\left(\xi^{0} \equiv 1\right),
$$

is that the form $A^{i}\left(x^{1}, \cdots, x^{n}\right) \delta x^{i}$ be an invariant of (6) and that $A_{0}\left(x^{1}, \cdots, x^{n}\right)$ be a scalar invariant of (6).

An application of Theorem 2 to the case in hand yields the conditions

$$
\left(\frac{\partial A_{\alpha}}{\partial x^{\beta}}-\frac{\partial A_{\beta}}{\partial x^{\alpha}}\right) \xi^{\beta}+\frac{\partial\left(A_{\sigma} \xi^{\sigma}\right)}{\partial x^{\alpha}}=0 .
$$

Since $A_{\alpha}$ and $\xi^{\alpha}$ are independent of $x^{0}$ and $\xi^{0} \equiv 1$, it follows by a simple calculation that the conditions (16) split up into the conditions (7) and

$$
\frac{\partial A_{0}}{\partial x^{j}} \xi^{j}=0
$$


But (7) are the conditions for the invariance of $A_{i} \delta x^{i}$ under the group (6) while (17) is, by a well known theorem of Lie, a necessary and sufficient condition that $A_{0}\left(x^{1}, \cdots, x^{n}\right)$ be a scalar invariant of the group (6).

THEOREM 5. A necessary and sufficient condition that the projective differential form (14) be an invariant attached to the path curves of the group (15) is that $A_{i} \delta x^{i}$ be an invariant of (6) and that

$$
A_{0}\left(x^{1}, \cdots, x^{n}\right)=-A_{k} \xi^{k} .
$$

With the aid of Theorem 3 we obtain the necessary and sufficient conditions

$$
\left(\frac{\partial A_{\alpha}}{\partial x^{\beta}}-\frac{\partial A_{\beta}}{\partial x^{\alpha}}\right) \xi^{\beta}=0, A_{\sigma} \xi^{\sigma}=0 .
$$

The first set of equations in (19) yields the conditions

$$
\left(\frac{\partial A_{i}}{\partial x^{j}}-\frac{\partial A_{j}}{\partial x^{i}}\right) \xi^{j}=\frac{\partial A_{0}}{\partial x^{i}}, \frac{\partial A_{0}}{\partial x^{j}} \xi^{j}=0
$$

while the last equation obviously is equivalent to

$$
A_{0}=-A_{k} \xi^{k} \text {. }
$$

But the equations (20) and (21) are completely equivalent to the conditions (7) and (21). This completes the proof of our theorem.

It is interesting to observe that the function $A_{0}$, as given by (18), is ipso facto a scalar invariant* of the group (6). Hence the attached invariant is distinguished from the unattached invariant (considered in Theorem 4 ) by the fact that not only must $A_{0}$ be a scalar invariant of the group (6) but it must have the particular form (18).

THEOREM 6. There always exist projective invariants of the types dealt with in Theorems $\dagger 4$ and 5 for any chosen one-parameter group appropriate to the case.

Cartan $\ddagger$ has given a number of interesting examples of projective invariants of the type considered in Theorem 5 . He did

* E. Goursat, loc. cit., p. 255.

$\dagger$ The same statement holds good for the invariants of Theorems 7 to 11 .

$\ddagger$ E. Cartan, loc. cit. 
not, however, give necessary and sufficient conditions nor did he write the conditions in an arbitrary coordinate system. Cartan referred to such invariants as complete integral invariants. Similar remarks hold good for the corresponding projective bilinear forms that are treated in the next paragraph.

\section{Alternating Integral Invariants.}

THEOREM 7. A necessary and sufficient condition that the projective differential form

$$
A_{\alpha \beta}\left(x^{1}, \cdots, x^{n}\right) \delta_{1} x^{\alpha} \delta_{2} x^{\beta}, \quad\left(A_{\alpha \beta}=-A_{\beta a}\right),
$$

be an invariant of the group (15) is that both $A_{i j} \delta_{1} x^{i} \delta_{2} x^{i}$ and $A_{0 j} \delta x^{i}$ be invariants of the group (6).

With the aid of the second part of Theorem 2 we see that necessary and sufficient conditions for the invariance of (22) are given by the system of differential equations

$$
\left(\frac{\partial A_{\alpha \beta}}{\partial x^{\gamma}}-\frac{\partial A_{\gamma \beta}}{\partial x^{\alpha}}-\frac{\partial A_{\alpha \gamma}}{\partial x^{\beta}}\right) \xi^{\gamma}+\frac{\partial\left(A_{\alpha \lambda} \xi^{\lambda}\right)}{\partial x^{\beta}}-\frac{\partial\left(A_{\beta \lambda} \xi^{\lambda}\right)}{\partial x^{\alpha}}=0 .
$$

For $\alpha=i, \beta=j$, these equations reduce to

$$
\left(\frac{\partial A_{i j}}{\partial x^{k}}-\frac{\partial A_{k j}}{\partial x^{i}}-\frac{\partial A_{i k}}{\partial x^{j}}\right) \xi^{k}+\frac{\partial\left(A_{i l} \xi^{l}\right)}{\partial x^{j}}-\frac{\partial\left(A_{j l} \xi^{l}\right)}{\partial x^{i}}=0,
$$

while for $\alpha=0, \beta=j$, they reduce to

$$
\left(\frac{\partial A_{0 j}}{\partial x^{k}}-\frac{\partial A_{0 k}}{\partial x^{j}}\right) \xi^{k}+\frac{\partial\left(A_{0 k} \xi^{k}\right)}{\partial x^{j}}=0 .
$$

The remaining equations in (23) are automatically satisfied on the basis of (24) and (25). Hence our theorem follows by an application of Theorem 2 .

THEOREM 8. A necessary and sufficient condition that the projective form (22) be an invariant attached to the path curves of the one-parameter group (15) is that $A_{i j} \delta_{1} x^{i} \delta_{2} x^{j}$ be an invariant of the group (6) and that $A_{i 0}\left(x^{1}, \cdots, x^{n}\right)$ be given by

$$
A_{i 0}=-A_{i k} \xi^{k} .
$$

With the aid of the second part of Theorem 3, we obtain the necessary and sufficient conditions 


$$
\left(\frac{\partial A_{\alpha \beta}}{\partial x^{\gamma}}-\frac{\partial A_{\gamma \beta}}{\partial x^{\alpha}}-\frac{\partial A_{\alpha \gamma}}{\partial x^{\beta}}\right) \xi^{\gamma}=0, \quad A_{\alpha \lambda} \xi^{\lambda}=0 .
$$

As can be readily verified, these equations split up into the following set of equations

$$
\left\{\begin{array}{l}
\left(\frac{\partial A_{i j}}{\partial x^{k}}-\frac{\partial A_{k j}}{\partial x^{i}}-\frac{\partial A_{i k}}{\partial x^{j}}\right) \xi^{k}=\frac{\partial A_{i 0}}{\partial x^{j}}-\frac{\partial A_{j 0}}{\partial x^{i}} \\
\left(\frac{\partial A_{0 j}}{\partial x^{k}}-\frac{\partial A_{0 k}}{\partial x^{j}}\right) \xi^{k}=0 \\
A_{i 0}=-A_{i k} \xi^{k}, \quad A_{0 k} \xi^{k}=0 .
\end{array}\right.
$$

The second set of equations is automatically satisfied on the basis of the first set and the last equation on the basis of the third set. Hence (28) is completely equivalent to the system of equations (9) and (26). This proves our theorem.

Definition. A linear differential form $\omega_{\delta}$ will be said to be a relative integral invariant of a one-parameter group appropriate to the case whenever the bilinear covariant $\delta_{2} \omega_{\delta_{1}}-\delta_{1} \omega_{\delta_{2}}$ is an invariant of the same one-parameter group.

Theorem 9. A necessary and sufficient condition that $A_{\alpha} \delta x^{\alpha}$ be a relative projective integral invariant of the group (15) is that $A_{i} \delta x^{i}$ be a relative integral invariant of (6) and that $A_{0}$ satisfy the differential equation

$$
\frac{\partial A_{0}}{\partial x^{k}} \xi^{k}=K,
$$

where $K$ is any chosen constant.

In fact, with the aid of Theorem 7 one can deduce without much difficulty the necessary and sufficient conditions

$$
\left\{\begin{array}{l}
\frac{\partial\left(A_{i k} \xi^{k}\right)}{\partial x^{j}}-\frac{\partial\left(A_{j k} \xi^{k}\right)}{\partial x^{i}}=0 \\
\left(\frac{\partial A_{0 j}}{\partial x^{k}}-\frac{\partial A_{0 k}}{\partial x^{j}}\right) \xi^{k}+\frac{\partial\left(A_{0 k} \xi^{k}\right)}{\partial x^{j}}=0,
\end{array}\right.
$$

where $A_{\alpha \beta}=\partial A_{\alpha} / \partial x^{\beta}-\partial A_{\beta} / \partial x^{\alpha}$. Now the second set of equations (30) reduces to 


$$
\frac{\partial}{\partial x^{j}}\left(\frac{\partial A_{0}}{\partial x^{k}} \xi^{k}\right)=0
$$

which proves our theorem.

Theorem 10. A necessary and sufficient condition that $A_{\alpha} \delta x^{\alpha}$ be a relative projective invariant attached to the path curves of the group (15) is that*

(a) the Pfaffian equation

$$
d A_{0}=\left(\frac{\partial A_{i}}{\partial x^{j}}-\frac{\partial A_{j}}{\partial x^{i}}\right) \xi^{j} d x^{i}
$$

be completely integrable;

(b) $A_{0}$ satisfy the equation (31).

We leave the details of proof to the reader for lack of space.

6. Concluding Remarks. The preceding paragraph was concerned with alternating projective differential forms and the reader may now wonder whether there are corresponding theorems for symmetric projective forms

$$
g_{\alpha \beta}\left(x^{1}, \cdots, x^{n}\right) \delta x^{\alpha} \delta x^{\beta}, \quad\left(g_{\alpha \beta}=g_{\beta \alpha}\right) .
$$

There is a partial negative answer to this query, for there does not exist a non-degenerate projective form (32) (that is, a form with a non-vanishing discriminant) attached to the path curves of the group (15). However, the following theorem holds good.

Theorem 11. A necessary and sufficient condition that (32) be a projective invariant of the group (15) is that $g_{i j} \delta x^{i} \delta x^{j}$ and $g_{i 0} \delta x^{i}$ be invariants of the group (6) and that $g_{00}$ be a scalar invariant of (6).

California Institute of Technology

* As an interesting application we observe that for the case of the Hamilton canonical equations, the Hamiltonian $H(q, p)$ is the unique function, up to an additive constant, in the relative invariant $p_{i} \delta q+A_{0} \delta t$. 